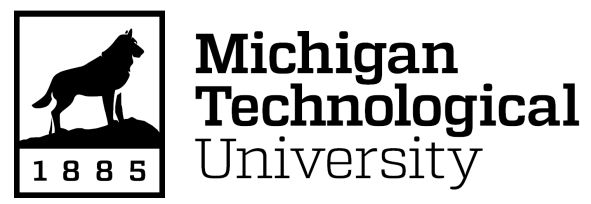

Michigan Technological University Digital Commons @ Michigan Tech

\title{
First-principles study of the variation of electron transport in a single molecular junction with the length of the molecular wire
}

\author{
Partha Pratim \\ Michigan Technological University \\ Ranjit Pati \\ Michigan Technological University
}

Follow this and additional works at: https://digitalcommons.mtu.edu/physics-fp

Part of the Physics Commons

\section{Recommended Citation}

Pratim, P., \& Pati, R. (2010). First-principles study of the variation of electron transport in a single molecular junction with the length of the molecular wire. Physical Review B, 82. http://dx.doi.org/10.1103/ PhysRevB.82.045424

Retrieved from: https://digitalcommons.mtu.edu/physics-fp/119

Follow this and additional works at: https://digitalcommons.mtu.edu/physics-fp

Part of the Physics Commons 


\title{
First-principles study of the variation of electron transport in a single molecular junction with the length of the molecular wire
}

\author{
Partha Pratim Pal and Ranjit Pati* \\ Department of Physics, Michigan Technological University, Houghton, Michigan 49931, USA \\ (Received 23 March 2010; revised manuscript received 30 June 2010; published 22 July 2010)
}

\begin{abstract}
We report a first-principles study of quantum transport in a prototype two-terminal device consisting of a molecular nanowire acting as an inter-connect between two gold electrodes. The wire is composed of a series of bicyclo[1.1.1]pentane (BCP) cage-units. The length of the wire $(L)$ is increased by sequentially increasing the number of BCP cage units in the wire from 1 to 3. A two terminal model device is made out of each of the three wires. A parameter free, nonequilibrium Green's function approach, in which the bias effect is explicitly included within a many body framework, is used to calculate the current-voltage characteristics of each of the devices. In the low bias regime that is considered in our study, the molecular devices are found to exhibit Ohmic behavior with resistances of $0.12,1.4$, and $6.5 \mu \Omega$ for the wires containing one, two, and three cages respectively. Thus the conductance value, $G_{c}$, which is the reciprocal of resistance, decreases as $e^{-\beta L}$ with a decay constant $(\beta)$ of $0.59 \AA^{-1}$. This observed variation of conductance with the length of the wire is in excellent agreement with the earlier reported exponential decay feature of the electron transfer rate predicted from the electron transfer coupling matrix values obtained using the two-state Marcus-Hush model and the Koopman's theorem approximation. The downright suppression of the computed electrical current for a bias up to $0.4 \mathrm{~V}$ in the longest wire can be exploited in designing a three terminal molecular transistor; this molecular wire could potentially be used as a throttle to avoid leakage gate current.
\end{abstract}

DOI: 10.1103/PhysRevB.82.045424

PACS number(s): 73.63.-b, 85.65.+h, 73.50.Fq, 71.10.-w

\section{INTRODUCTION}

Inter/intramolecular electron/hole transfer has been a fascinating topic of research since the last few decades. ${ }^{1}$ The obvious reason behind this upsurge in research interest being the continuous desire to better understand the natural biological processes. In nature, each and every chemical/ biological phenomenon is dictated by the interaction of several molecules. How do these molecules interact between themselves? Electron is the agent, and the transfer of electrons from one molecule to another is one of the dominant modes of signal the molecules use to communicate. ${ }^{2}$ In this perspective, controlled electron transfer would be an important subject to unearth. Another driving force behind the study of electron transfer in molecules is the craze for miniaturizing electronic devices where the "toy idea" of building electronic devices with molecules would be a phenomenal solution. ${ }^{3}$ In fact, electron transport measurements in single molecular junctions have been reported successfully by several research groups; ${ }^{4-14}$ for a comprehensive review see Refs. 15-18. These premier experiments have generated significant interest toward understanding the underlying physics behind the current-voltage characteristics across molecular nanojunctions. ${ }^{4}$

The next question would be: "What kind of molecules would one choose to study electron transfer?" A certain category of organic molecules known as rigid molecular rods ${ }^{19}$ can be considered to be good candidates for studying electron transport owing to their stability in adverse conditions. The name 'rod' is actually deceptive as they are very flexible ${ }^{19}$ and are not easily distorted. One should not mistake them to be tough iron rods used in our daily life. In fact, they can be thought of as soft elastic rods, which can withstand distortions whether externally or internally and still retain their electronic properties. It is due to this property that they can act as mediators or interconnects in between two active centers/units without getting their own electronic structure reorganized. One also finds them to be useful in the construction of big molecules and supramolecular assemblies. ${ }^{19}$

Bicyclo[1.1.1]pentane (BCP) is one of the smallest synthesized 'rigid molecular rods'. Producing ${ }^{20}$ and storing this molecule is quite simple. They are stable in temperatures up to $280-300{ }^{\circ} \mathrm{C}$ and are transparent in the UV spectrum up to $200 \mathrm{~nm} .{ }^{19}$ Several groups have already reported electron transfer coupling matrix $\left(V_{A B}\right)$ calculations for this system $^{21-23}$ using Koopman's theorem approximation and Marcus-Hush two state model ${ }^{24-26}$ within Hartree-Fock (HF) theory. However, to understand the current-voltage characteristics of this molecular wire, one needs to consider a device geometry where the finite molecule is attached in between two semi-infinite electrodes used as a source and a drain. In such an open and heterogeneous system, applied bias drives the electron from source to drain; it is a non-equilibrium process. Modeling such a process in a heterogeneous system is a challenging task as it depends not only on the intrinsic properties such as geometry, conformation, length, electronic, and magnetic structure of the molecular system but also on the electronic structure at the molecule-lead interface. Here, we report modeling of such a process in a prototypical metal-molecule junction. A two-terminal prototype device made out of this molecule would contain a BCP molecule sandwiched between two semi-infinite metallic electrodes. Bulk Au or Pt are the most commonly used electrode materials. It has been observed that the presence of an anchoring group facilitates the formation of a metal-molecule bond. Studies on the effect of various anchoring groups on the binding strength of metal-molecule junctions reveal that 
thiol (-SH) end groups along with $\mathrm{Au}$ electrodes form the most stable junction. ${ }^{12,27,28}$ Such is the strength of the S-Au bond that the stretching of the metal-molecule junction actually results in stretching of $\mathrm{Au}-\mathrm{Au}$ bonds. This creates a $\mathrm{Au}$ nanowire before breaking the $\mathrm{Au}-\mathrm{Au}$ bond. This was first predicted by molecular dynamics (MD) calculations ${ }^{29}$ and was later observed experimentally. ${ }^{30}$ With these strong evidences, we choose thiolate(-S) to be the anchoring group and $\mathrm{Au}$ as the electrode.

We then proceed to study the current-voltage characteristics of this model device using a parameter free, nonequilibrium Green's function (NEGF) formalism. A first-principles density functional method with Becke's three parameter hybrid functional ${ }^{31}$ is used to construct the nonequilibrium Green's function; the bias effect is explicitly included in our approach within a many-body framework. The calculated current-voltage characteristic in the device shows an Ohmic behavior in a low-bias regime. An increase in the length of the BCP molecular wire results in an exponential decay in the magnitude of conductance, which is in excellent agreement with the exponential decay feature ${ }^{21,23}$ of the electron transfer rate predicted from the length dependent trend of electron transfer coupling matrix value, $V_{A B}$. The decay constant $(\beta)$ is calculated to be $0.59 \AA^{-1}$. It is found that by increasing the number of $\mathrm{BCP}$ cage units from 1 to 3 in the wire, one can completely suppress the current in the molecular device for a bias up to $0.4 \mathrm{~V}$. This suggests that this wire could potentially be used as a gate throttle to avoid leakage gate current in a three terminal junction.

The remainder of this paper is organized as follows. In Sec. II, the modeling of the device is described. The results of this work are discussed extensively in Secs. III and IV briefly summarize our main findings.

\section{MODELING THE DEVICE}

A prototypical molecular device that consists of a finitesized molecule slotted in between two semi-infinite metallic electrodes is an open system. To model such a heterogeneous system, we have divided it into two parts. One is the active scattering region, which consists of a molecule sandwiched in between two clusters comprising a finite number of electrode atoms that are perturbed when the molecule adsorbs onto the electrode surface. The rationale behind the choice of a finite number of electrode atoms in the active region of the device is that the adsorption of the molecule on the electrode results in a local-charge screening within the metallic electrode at the interface. ${ }^{32,33}$ The single electron energy levels in this finite region are calculated using finite-cluster densityfunctional theory (DFT). The second part of the device consists of the rest of the electrode on each side and is considered to retain its bulk properties. Thus, this part is considered to be the unlimited "source" for electrons on one side and the "sink" on the other side in the nonequilibrium condition. The Hamiltonian in the active device region that takes the bias effect explicitly ${ }^{34}$ into account is:

$$
H(\varepsilon)=H(0)+\vec{\varepsilon} \cdot \sum_{i} \vec{r}(i),
$$

where $H(0)$ is the unperturbed Hamiltonian; $\vec{\varepsilon}$ is the applied dipole electric field along the wire axis, and $\vec{r}(i)$ is the coor- dinate of the $i$ th electron. A real space approach in which the many-body wave function of the electron is expanded in terms of a finite set of Gaussian atomic orbitals ${ }^{35}$ is adopted to calculate $H(\varepsilon)$. In our calculation we have used a posteriori Becke's three parameter hybrid functional (B3LYP) ${ }^{31}$ that includes a part of the exact HF exchange and the LeeYang-Parr (LYP) functional, which ensures the inclusion of local and nonlocal correlation terms. ${ }^{35,36}$ The inclusion of a part of the exact HF exchange, calculated using the Slater determinant of the Kohn-Sham orbitals, ${ }^{31}$ allows us to avoid the self-interaction errors that occur in conventional DFT. The Los Alamos double zeta effective core potential (LANL2DZ) basis set $^{35}$ that includes the scalar relativistic effects is used for the electrode $(\mathrm{Au})$ atoms. A triple valence zeta Gaussian basis function with an additional polarization function on heavy atoms $\left(6-311 G^{*}\right)$ is used for the atoms of the molecule. To ensure tight convergence during selfconsistent calculations, the convergence criterion for energy, maximum, and root mean-square electron density are set at $10^{-6}, 10^{-6}$, and $10^{-8}$ a.u., respectively. The use of real space approach allows us to partition the $H(\varepsilon)$ to obtain the Hamiltonian corresponding to the molecular part as well as the molecule-lead (only the perturbed atoms of the electrode) coupling matrices.

NEGF, in its various forms,${ }^{37-41}$ provides the perfect tool to "couple" the finite and the bulk portion of the model device. A brief overview of the NEGF method that is employed to study the transport characteristics of the device is given below. The primary quantity of the formalism is the Green's function, which in our case has an implicit bias dependence through $\varepsilon$ and is calculated as:

$$
G(E, \varepsilon)=\left[E \times S-H_{m o l}(\varepsilon)-\Sigma_{l}(\varepsilon)-\Sigma_{r}(\varepsilon)\right]^{-1} .
$$

$E$ is the injection energy of the tunneling electron, $H_{m o l}(\varepsilon)$ is the molecular part of the orthogonalized Kohn-Sham Hamiltonian matrix obtained from $H(\varepsilon)$, and $S$ is an identity matrix. $\Sigma_{l, r}(\varepsilon)$ are the bias-dependent self-energy functions, ${ }^{42,43}$ which depict the molecule-electrode interactions. The expressions for $\Sigma_{l, r}(\varepsilon)$ are:

$$
\Sigma_{l, r}(\varepsilon)=C_{l, r}^{\dagger} G_{p}(E) C_{l, r},
$$

where $C_{l, r}$ are the orthogonalized bias-dependent moleculelead coupling matrices. Thus, in our calculations the nonequilibrium nature of the electronic coupling between the molecule and the electrode is accounted for at each bias value; structural reorganization under bias is not considered. It is important to note that under nonequilibrium situation (i.e., in the presence of applied bias), structural arrangements at the molecule-lead interface cannot be expected to be the same as those under the equilibrium condition. However, for a strongly coupled molecular junction that is considered here, the mass-scale rearrangement of atoms at the interfacial geometry is not expected to occur. $G_{p}$, the Green's function of the lead $(\mathrm{Au})$, is an implicit function of injection energy $E$; it is defined as:

$$
G_{p}(E)=-i \pi \eta(E) \times I_{m} .
$$

$I_{m}$ is an identity matrix of dimension $m \times m ; m$ is the total number of Gaussian basis functions used to represent the $\mathrm{Au}$ 
atoms in the active region of the device. $\eta(E)$ is obtained from the density of states (DOS) of the bulk Au, which is calculated using the periodic DFT implemented within the VASP code. ${ }^{44}$ For this calculation, we have used plane wave basis function and density functional theory within generalized gradient approximation for the exchange-correlation. The valence-core interaction is described by the projectoraugmented wave (PAW) approach. The optimized lattice parameter for the fcc gold is found to be $4.175 \AA$. To calculate the DOS, we have used $51 \times 51 \times 51 k$-point sampling within the Monkhorst-Pack scheme to sample the Brillouin zone. The robustness of the $k$-point sampling is tested by comparing the energy of the system for different $k$-points (the energy convergence criterion with respect to the choice of $k$-points was less than $0.001 \mathrm{eV})$. The value for $\eta(\mathrm{E})$ is calculated as DOS (E) per electron in the unit cell. The energy grid $(0.002 \mathrm{eV})$ is taken as the same grid used for the integration in Eq. (7). Fermi energy of the bulk gold is aligned with the Fermi level of the active region of the device at equilibrium $(V=0)$. The probability of electrons pumped into the system from the source reaching the drain is determined by the bias-dependent transmission function, which is a sum of the transmission probabilities over all conducting channels, and is calculated as: ${ }^{42,43}$

$$
T_{l r}=\operatorname{Tr}\left[\Gamma_{l} G \Gamma_{r} G^{\dagger}\right],
$$

where $\Gamma_{l, r}$ are the broadening functions, which determine the escape rate of electrons; they are given by:

$$
\Gamma_{l, r}=i\left[\Sigma_{l, r}(\varepsilon)-\Sigma_{l, r}^{\dagger}(\varepsilon)\right] \text {. }
$$

Finally, within the coherent scattering approximation, the current through the molecular junction is calculated using the multichannel Landauer-Büttiker formalism. ${ }^{42,43,45,46}$ In this approach, the expression for the current is:

$$
I=\frac{2 e}{h} \int_{\mu_{1}}^{\mu_{2}} T_{l r}\left[f\left(E, \mu_{2}\right)-f\left(E, \mu_{1}\right)\right] d E,
$$

where $f$ is the Fermi distribution function, $e$ is the electronic charge, $h$ is the Planck's constant. $\mu_{1,2}$ are calculated as:

$$
\mu_{1,2}=E_{f} \mp V_{\text {low,high }},
$$

The $V_{\text {low,high }}$ is calculated self-consistently for each applied $\varepsilon$ using the difference between the electrostatic potentials ${ }^{47}$ at finite and zero bias at the two metal-molecule junctions; $E_{f}$ is the equilibrium Fermi energy. The potential difference, $V$, is obtained from the difference of $V_{\text {low }}$ and $V_{\text {high }}$ for each applied $\varepsilon$. It should be noted that at equilibrium (zero bias) $V_{\text {low }}=V_{\text {high }}$ An additional thermal smearing term, $k_{B} T(=0.026 \mathrm{eV})$, is subtracted from $\mu_{1}$ and added to $\mu_{2}$ to take into account the electronic temperature at the contact in the nonequilibrium condition; it essentially increases the integration window width by an additional constant of 0.052 $\mathrm{eV}$ for each applied bias.

\section{RESULTS AND DISCUSSIONS}

\section{A. Device structure}

As mentioned earlier, we have constructed a BCP twoterminal molecular device by sandwiching a molecular moi-

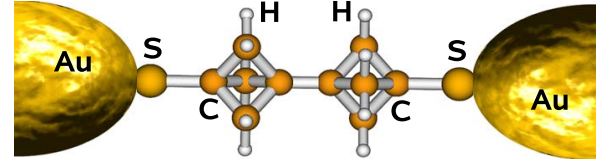

FIG. 1. (Color online) Schematic of a two-terminal device built out of a molecular wire containing two BCP cages.

ety between two Au electrodes with thiolate as the anchoring group. We have calculated the bias driven current for the three different wires containing 1, 2, and 3 BCP cages. A prototype two-terminal device built out of a molecular wire containing two BCP cages is shown in Fig. 1. Though the structure of the free BCP molecule is known, ${ }^{19,23}$ the atomic level structural details of the contact geometry at the molecule-gold interface is unknown. Hence, we first optimize each of the molecular wires with thiol $(-\mathrm{SH})$ as the anchoring group using DFT within B3LYP formalism; Gaussian electronic structure code is used $^{35}$ for the optimization. The distance between the terminal $\mathrm{C}$ atom and the $\mathrm{S}$ atom in the thiol group to which it is bonded is found to be $1.8 \AA$. The distance between the two terminal $\mathrm{C}$ atoms is $1.86 \AA$, which is in good agreement with the previously reported value of $1.87 \AA .{ }^{19,23}$ Subsequently, the optimized molecular structure with thiolate $(-\mathrm{S})$ as the anchoring group is embedded between two clusters of 3-Au atoms on each side to model the active region. The $\mathrm{S}$ atom is incorporated into the threefold hollow site of the $\mathrm{Au}$ atoms on the $\mathrm{Au}(111)$ surface. ${ }^{48}$ It should be noted that for practical purposes, we have considered only three gold atoms on each side which are directly involved in bonding with the $\mathrm{S}$ atoms. The distance between the $\mathrm{Au}$ and $\mathrm{S}$ is varied to determine the minimum energy configuration. The optimized S-Au distance is found to be $2.80 \AA$; to discern the role of the molecular spacer on conductance, the interfacial contact geometry is kept the same for all the three molecular devices.

\section{B. Potential profile}

To understand the response of the molecule to external bias, we applied a dipole electric field along the molecular wire axis as discussed in Sec. II. The electrostatic potential is then calculated self-consistently ${ }^{47}$ at each atomic center in the active region for each applied field as well as for the zero bias. The difference between the electrostatic potentials at $\varepsilon=0(V=0)$ and $\varepsilon \neq 0$ is then obtained at each atomic center, which is subsequently averaged over the degrees of freedom perpendicular to the wire axis to obtain the relative electrostatic potential (REP) profile along the wire axis. The REP values are then plotted along the molecular wire axis for three different externally applied fields (Fig. 2). For brevity, we have only presented the results for a molecular wire containing two BCP cages in Fig. 2.

The magnitude of REP at the molecule-lead interface increases with the increase of applied field as expected (Fig. 2). For example, at an external applied field of $0.11 \mathrm{~V} / \AA$, the REP at the left and right junction is $0.19 \mathrm{~V}$ and $-0.19 \mathrm{~V}$ respectively. For an applied field of $0.03 \mathrm{~V} / \AA$, the respective REPs are $0.05 \mathrm{~V}$ and $-0.05 \mathrm{~V}$. However, an intriguing 


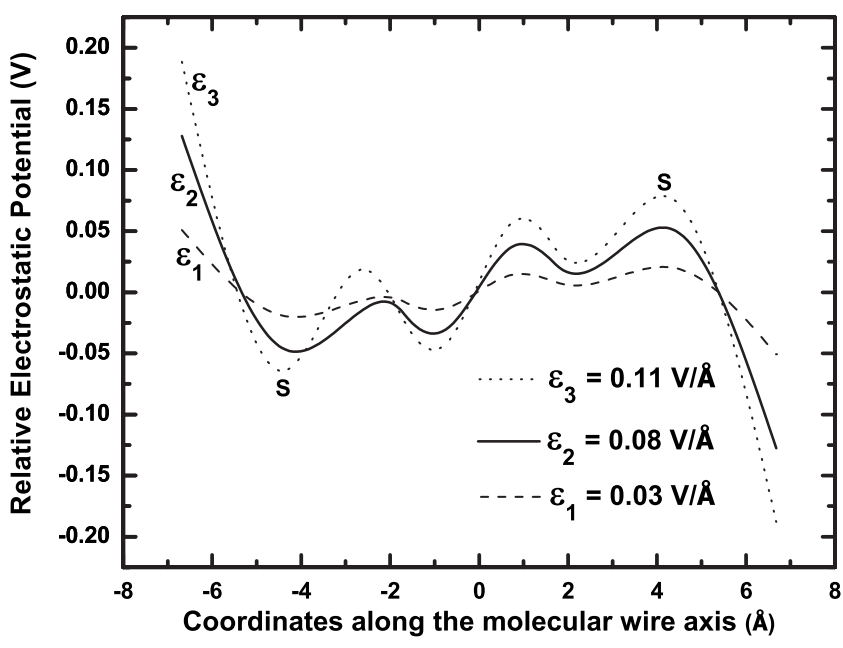

FIG. 2. Potential profile of the two-cage molecular wire for different electric fields, $\varepsilon_{1}, \varepsilon_{2}$, and $\varepsilon_{3}$. The notation " $S$ " refers to the position of the terminal $\mathrm{S}$ atoms in the device.

feature is revealed by examining the potential profile between the two terminal S-atoms; one can clearly notice two effective potential barriers. Furthermore, the barrier height changes with the increase of applied field. This clearly suggests that for quantitative evaluation of bias induced response in a molecular device, the explicit inclusion of field effect is essential. Next, we focus on the potential profile of the molecular wires containing one, two, and three BCP cages. The potential profiles at $V=0.24 \mathrm{~V}$ for the three molecular wires are encapsulated in Fig. 3. For the molecular wire containing one $\mathrm{BCP}$ cage, there is only one effective potential barrier between the two terminal S-atoms. For the wire with two and three BCP cages, two and three effective potential barriers are noticeable. This suggests that by increasing the number of BCP cage units in the wire, we are essentially increasing the number of effective potential barriers in the conducting molecular channels. Another interesting, noticeable feature from Fig. 3 is that the barrier height for the wire with a single $\mathrm{BCP}$ cage is relatively smaller than that of the wires containing two and three BCP cages. The height and the number of effective barriers have important implication on the quantum transport properties of these wires, which will be revisited in the next section. To account for the origin of valley and hill at the terminal S-atom position in the potential profiles (Figs. 2 and 3), we analyzed the variation of Mulliken charge associated with $\mathrm{S}$ atoms as a function of bias. For a wire containing a single BCP cage, the charge profile is presented in Fig. 4. At equilibrium (zero applied bias), both of the terminal $\mathrm{S}$ atoms have equal amounts of charge (scaled to zero in the charge scale in Fig. 4). As the bias increases, the negative charge on the left $S$ increases steadily; at the same time positive charge accumulation increases for the right $\mathrm{S}$ atom. This bias induced polarization effect is responsible for the hill and valley observed in the potential profile. Similar bias induced polarization effects were noted for wires containing two, and three $\mathrm{BCP}$ units.

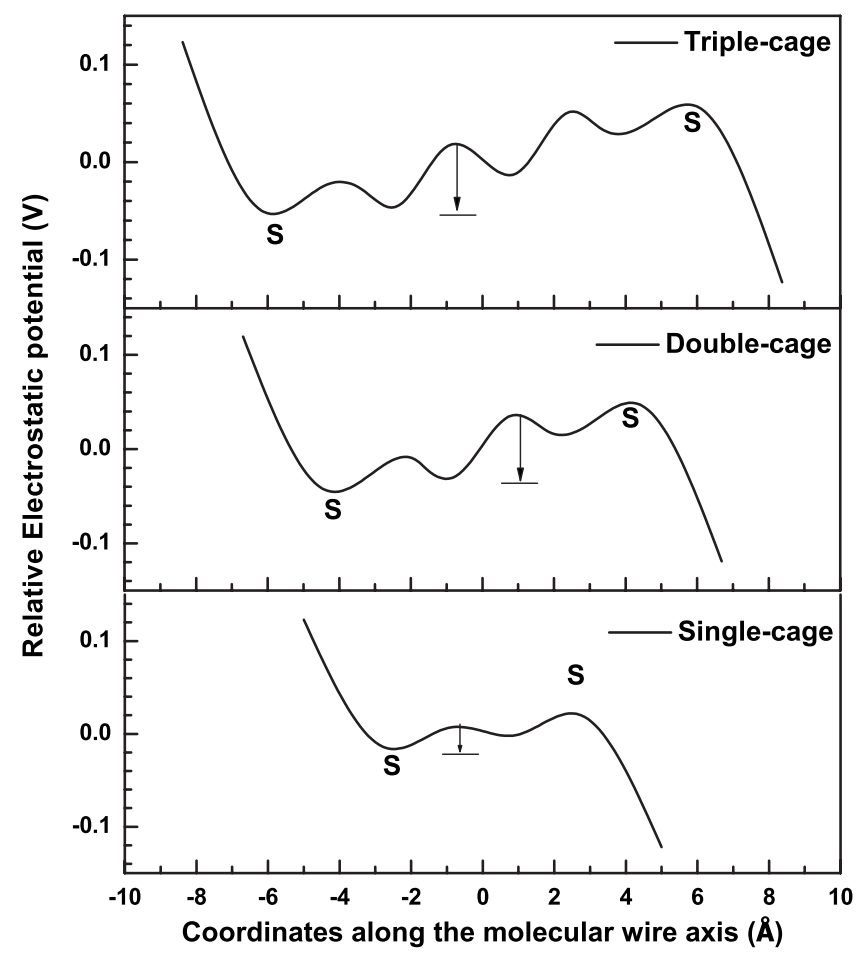

FIG. 3. Potential profile of the three different wires at $V=0.24$ Volts. The notation " $\mathrm{S}$ " refers to the position of the terminal $\mathrm{S}$ atoms in the device. The arrows indicate the height of the effective potential barriers.

\section{C. $I-V$ characteristics}

The calculated currents through the molecular wires as a function of external bias, $V$, are summarized in Fig. 5. For the low-bias regime considered in our calculation, the $I-V$ curves follow Ohm's Law, i.e., the current increases linearly with the bias, $V$. As expected, the wire containing the three $\mathrm{BCP}$ cage units has the largest resistance of the three and hence the least current for a particular bias. For example at $0.41 \mathrm{~V}$, the current in the single-cage wire is $3.4 \mu \mathrm{A}$; the current in the double-cage wire is $0.32 \mu \mathrm{A}$; and the current

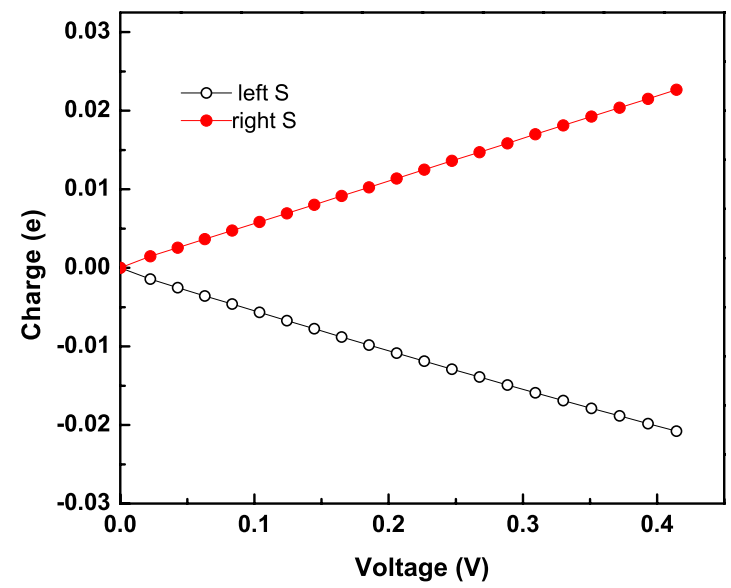

FIG. 4. (Color online) Charge profile depicting bias induced polarization effect on the terminal " $\mathrm{S}$ " atoms in the molecular wire containing one BCP cage unit. 


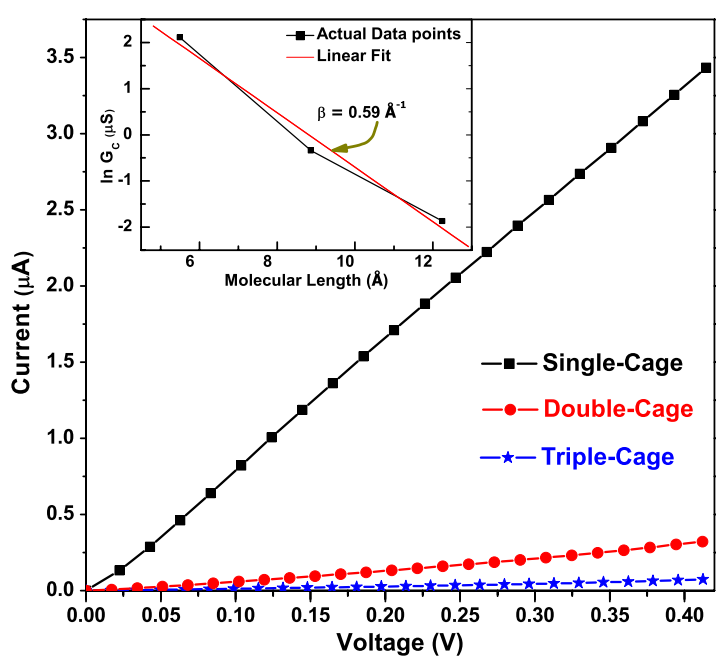

FIG. 5. (Color online) $I-V$ characteristics of the three different wires under the influence of applied external bias. Inset shows the plot of $\ln \left(G_{c}\right)$ vs length of the molecule $(l)$. A straight line is fitted into the data points in order to calculate $\beta$.

in the triple cage wire is $0.07 \mu \mathrm{A}$. The resistance of the three wires calculated from the slopes of the $I-V$ curves are $0.12 \mu \Omega, 1.4 \mu \Omega, 6.5 \mu \Omega$ for the one-cage, two-cage, and three-cage wire, respectively. The substantial increase in resistance or the decay in current with the increase in the number of cage units in the wire can also be understood from Fig. 3 . The increase in the number of barriers with the increase in the number of cage units in the wire is responsible for the decrease in the current. From one-cage unit to two-cage unit in the wire, the number of effective potential barriers in between the terminal S-atoms increases to two. In addition, the effective barrier heights also increase leading to a significant drop in current. Going from two-cage to three-cage unit in the wire, the number of effective potential barriers between the terminal S-atoms increases from two to three. However, the barrier height does not show a significant change. This explains why only a small drop in current is observed when increasing the number of cage-units from two to three. The conductance values, obtained from the inverse of resistances, are found to decrease exponentially with the increase of the number of cage-units in the wire. It follows a simple relation:

$$
G_{c}[L]=G_{c}(L=0) e^{-\beta L},
$$

where $\beta$ is a decay constant associated with the decrease in the conductance value as the length of the molecular wire $(L)$ is increased; $G_{c}(L=0)$ is the extrapolated conductance at the interface $(L=0)$. This exponential decay feature of the conductance is in excellent agreement with the length-dependent decay feature in the electron transfer rate predicted from the electron transfer coupling matrix values, which re-affirm the accuracy of our theoretical approach. It should be noted that the conductance value is proportional to the electron transfer rate, and hence $V_{A B}$. In the latter case the $V_{A B}$ was calculated using the two-state Marcus-Hush model and the Koopman's theorem approximation; no bias effect was considered. The $\beta$ value reported from the Koopman's theorem approximation ${ }^{21}$ was $0.66 \AA^{-1}$; the two-state Marcus-Hush model yielded ${ }^{23}$

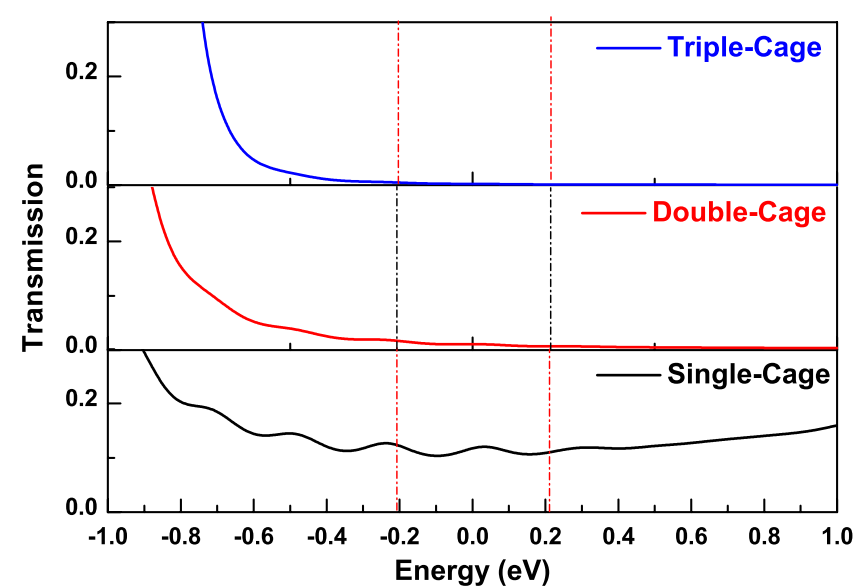

FIG. 6. (Color online) Transmission function as a function of injection energy in the three molecular wires under the same applied external voltage $(=0.41 \mathrm{~V})$. The chemical potential window is shown by the dotted line.

the $\beta$-value to be $0.97 \AA^{-1}$. To determine the $\beta$-value, we plotted the variation in $\ln \left(G_{c}\right)$ with the length of the wire $(L)$ as shown in the inset of Fig. 5. The distance between the terminal S-atoms is considered to be the length of the wire. The data points are fitted to a straight line to calculate the $\beta$ as $0.59 \AA^{-1}$. The discrepancy between the current $\beta$ value and the $\beta$ value obtained from $V_{A B}$ result could be ascribed to the fact that in the former case the bias effect was not explicitly considered. In addition, the electrode induced polarization effect on the molecular orbital was not taken into account during the calculation of $V_{A B}$.

\section{Transmission function}

To gain a deeper insight into the $I$ - $V$ characteristics of this particular metal-molecular wire-metal system, we look into the entities that determine the electrical current through the junction, precisely the bias dependent transmission function, $T_{l r}$ [Refer Eqs. (5) and (7)]. $T_{l r}$ as a function of injection energy $E$, is outlined in Fig. 6; a bias value of $0.41 \mathrm{~V}$ is considered. The chemical potential window $\left[\mu_{1}=-0.2, \mu_{2}\right.$ $=0.21]$ is shown by the dotted line; Fermi energy is set to zero in the energy scale. The transmission function looks fairly flat within the low-bias regime (0-0.41 Volts) considered in our calculation. $T_{l r}$ is devoid of spikes as seen in some other molecular species. ${ }^{43}$ A rugged $T_{l r}$ can give rise to nonlinear $I-V$ characteristics or even negative differential resistance (NDR). ${ }^{43}$ A relatively smooth $T_{l r}$ within the integration window is the possible reason behind the Ohmic behavior in this type of molecular wires. A comparative study of the $T_{l r}$ for the three devices within the same chemical potential window reveals a significantly higher transmission value for a single-cage wire when compared to the wire containing two cages. For instance at $0.1 \mathrm{eV}$ injection energy, the value of the transmission function for the single cage wire is 0.11 as compared to 0.008 for the two cage wire. In the triple cage system, $T_{l r}$ is 0.002 for the same injection energy. This clearly explains the length dependent $I-V$ features observed in Fig. 5. 


\section{CONCLUSIONS}

In summary, we have used first-principles density functional method with a posteriori B3LYP approach to study the quantum transport properties of BCP molecular devices. A parameter free, nonequilibrium Green's function approach, where the bias effect is explicitly included within a manybody formalism, is used to compute the current-voltage characteristics of the device. In the low bias regime considered in our study, we found the $I-V$ feature to follow Ohm's law. We estimated the resistance from the slope of the $I-V$ curve. By increasing the number of BCP units in the molecular wire, the conductance value is found to decrease exponentially with a decay constant, $\beta$, of $0.59 \AA^{-1}$. This is in excellent agreement with the exponential decay feature observed for the length-dependent electron transfer rate in the same system predicted from the two-state Marcus-Hush approach. By including three BCP units in the wire, we found that the current can be completely suppressed for a bias up to $0.41 \mathrm{~V}$. This suggests that the wire containing three $\mathrm{BCP}$ cage units could potentially be used as a gate throttle to avoid leakage gate current in a three terminal molecular transistor.

\section{ACKNOWLEDGMENT}

This work is supported by NSF through Grant No. ECCS0643420 .

*patir@mtu.edu

${ }^{1}$ C. L. Closs and J. R. Miller, Science 240, 440 (1988).

${ }^{2}$ A. Bandyopadhyay, R. Pati, S. Sahu, F. Peper, and D. Fujita, Nat. Phys. 6, 369 (2010).

${ }^{3}$ A. Aviram and M. A. Ratner, Chem. Phys. Lett. 29, 277 (1974).

${ }^{4}$ M. A. Reed, C. Zhou, C. J. Muller, T. P. Burgin, and J. M. Tour, Science 278, 252 (1997).

${ }^{5}$ X. D. Cui, A. Primak, X. Zarate, J. Tomfohr, O. F. Sankey, A. L. Moore, T. A. Moore, D. Gust, G. Harris, and S. M. Lindsay, Science 294, 571 (2001).

${ }^{6}$ J. Park, A. N. Pasupathy, J. I. Goldsmith, C. Chang, Y. Yaish, J. R. Petta, M. Rinkoski, J. P. Sethna, H. D. Abruna, P. L. McEuen, and D. C. Ralph, Nature (London) 417, 722 (2002).

${ }^{7}$ W. Liang, M. P. Shores, M. Bockrath, J. R. Long, and H. Park, Nature (London) 417, 725 (2002).

${ }^{8}$ B. Xu and N. J. Tao, Science 301, 1221 (2003).

${ }^{9}$ X. Xiao, B. Xu, and N. J. Tao, Nano Lett. 4, 267 (2004).

${ }^{10}$ X. Li, J. He, B. Xu, S. M. Lindsay, and N. J. Tao, J. Am. Chem. Soc. 128, 2135 (2006).

${ }^{11}$ M. Tsutsui, Y. Teramae, S. Kurokawa, and A. Sakai, Appl. Phys. Lett. 89, 163111 (2006).

${ }^{12}$ F. Chen, X. Li, J. Hihath, Z. Huang, and N. J. Tao, J. Am. Chem. Soc. 128, 15874 (2006)

${ }^{13}$ E. Lörtscher, H. B. Weber, and H. Riel, Phys. Rev. Lett. 98, 176807 (2007).

${ }^{14}$ H. Song, Y. Kim, Y. H. Jang, H. Jeong, M. A. Reed, and T. Lee, Nature (London) 462, 1039 (2009).

${ }^{15}$ C. Joachim, J. K. Gimzewski, and A. Aviram, Nature (London) 408, 541 (2000).

${ }^{16}$ A. Nitzan and M. A. Ratner, Science 300, 1384 (2003).

${ }^{17}$ J. R. Heath and M. A. Ratner, Phys. Today 56(5), 43 (2003).

${ }^{18}$ N. J. Tao, Nat. Nanotechnol. 1, 173 (2006).

${ }^{19}$ P. F. H. Schwab, M. D. Levin, and J. Michl, Chem. Rev. 99, 1863 (1999).

${ }^{20}$ K. B. Wiberg, D. S. Connor, and G. M. Lampman, Tetrahedron Lett. 5, 531 (1964).

${ }^{21}$ C. Liang and M. D. Newton, J. Phys. Chem. 97, 3199 (1993).

${ }^{22}$ R. Pati and S. P. Karna, J. Chem. Phys. 115, 1703 (2001).

${ }^{23}$ R. Pati and S. P. Karna, Chem. Phys. Lett. 351, 302 (2002).

${ }^{24}$ N. S. Hush, Electrochim. Acta 13, 1005 (1968).

${ }^{25}$ R. A. Marcus and N. Sutin, Biochim. Biophys. Acta 811, 265

(1985).

${ }^{26}$ M. D. Newton, Adv. Chem. Phys. 106, 303 (1999).

${ }^{27}$ Y. Xia and G. M. Whitesides, Adv. Mater. 7, 471 (1995).

${ }^{28}$ A. Ulman, Chem. Rev. 96, 1533 (1996).

${ }^{29}$ D. Krüger, H. Fuchs, R. Rousseau, D. Marx, and M. Parrinello, Phys. Rev. Lett. 89, 186402 (2002).

${ }^{30}$ Z. Huang, F. Chen, P. A. Bennett, and N. J. Tao, J. Am. Chem. Soc. 129, 13225 (2007).

${ }^{31}$ A. D. Becke, J. Chem. Phys. 98, 5648 (1993).

${ }^{32}$ A. R. Williams, P. J. Feibelman, and N. D. Lang, Phys. Rev. B 26, 5433 (1982).

${ }^{33}$ G. P. Brivio and M. I. Trioni, Rev. Mod. Phys. 71, 231 (1999).

${ }^{34}$ A. Szabo and N. S. Ostlund, Modern Quantum ChemistryIntroduction to Advanced Electronic Structure Theory (Dover, New York, 1996).

${ }^{35}$ GAUSSIAN 03, Gaussian Inc., Pittsburgh, PA, 2003.

${ }^{36}$ R. G. Parr and W. Yang, Density-Functional Theory of Atoms and Molecules (Oxford Science, Oxford, 1994).

${ }^{37}$ S. Datta, W. Tian, S. Hong, R. Reifenberger, J. I. Henderson, and C. P. Kubiak, Phys. Rev. Lett. 79, 2530 (1997).

${ }^{38}$ J. Taylor, H. Guo, and J. Wang, Phys. Rev. B 63, 245407 (2001).

${ }^{39}$ M. Brandbyge, J.-L. Mozos, P. Ordejón, J. Taylor, and K. Stokbro, Phys. Rev. B 65, 165401 (2002).

${ }^{40}$ K. S. Thygesen and A. Rubio, J. Chem. Phys. 126, 091101 (2007).

${ }^{41}$ G. C. Solomon, C. Harrmann, T. Hansen, V. Mujica, and M. A Ratner, Nat. Chem. 2, 223 (2010).

${ }^{42}$ S. Datta, Electron Transport in Mesoscopic Systems (Cambridge University Press, Cambridge, England, 1997).

${ }^{43}$ R. Pati, M. McClain, and A. Bandyopadhyay, Phys. Rev. Lett. 100, 246801 (2008)

${ }^{44}$ Vienna ab initio Simulation Package (VASP), Technische Universität Wien, 1999; G. Kresse and J. Furthmüller, Phys. Rev. B 54, 11169 (1996).

${ }^{45}$ Y. Meir and N. S. Wingreen, Phys. Rev. Lett. 68, 2512 (1992).

${ }^{46}$ R. Pati, L. Senapati, P. M. Ajayan, and S. K. Nayak, Phys. Rev. B 68, 100407(R)(2003).

${ }^{47}$ B. G. Johnson, P. M. W. Gill, J. A. Pople, and D. J. Fox, Chem. Phys. Lett. 206, 239 (1993).

${ }^{48}$ J. G. Zhou and F. Hagelberg, Phys. Rev. Lett. 97, 045505 (2006). 